\title{
Tracking and segmentation using min-cut with consecutive shape priors
}

\begin{abstract}
Junqiu Wang* ${ }^{*}$, Abstract
Yasushi Yagi ${ }^{\dagger}$

The institute of Scientific and Industrial Research, Osaka University, 8-1 Mihogaoka Ibaraki, Osaka, 560-0047

Received 25 November 2009 Accepted 19 March 2010

Tracking and segmentation find a wide range of applications such as intelligent sensing of robots, human-computer interaction, and video surveillance. Tracking and segmentation, however, are challenging for many reasons, e.g., complicated object shapes, cluttered background. We propose a tracking and segmentation algorithm that employs shape priors in a consecutive way. We found that shape information obtained using the Min-Cut algorithm can be applied in segmenting the consecutive frames. In our algorithm, the tracking and segmentation are carried out consecutively. We use an adaptive tracker that employs color and shape features. The target is modeled based on discriminative features selected using foreground/background contrast analysis. Tracking provides overall motion of the target for the segmentation module. Based on the overall motion, we segment object out using the effective min-cut algorithm. Markov Random Fields, which are the foundation of the min-cut algorithm, provide poor priors for specific shapes. It is necessary to embed shape priors into the min-cut algorithm to achieve reasonable segmentation results. Object shapes obtained by segmentation are employed as shape priors to improve segmentation in next frame. We have verified the proposed approach and got positive results on challenging video sequences. $\$$
\end{abstract}

\section{Keywords}

tracking $\cdot$ segmentation $\cdot$ mean-shift $\cdot$ min-cut $\cdot$ shape priors $\cdot$ consecutive shape matching

\section{Introduction}

Tracking and segmentation find applications in a wide range of fields, e.g., human-computer interface, video surveillance, and intelligent robotics. There are three common steps in these tasks: detecting moving objects such as pedestrians, tracking and segmenting objects from frame to frame, and behavior analysis based on segmented objects. The goal of our work is to track and segment objects in video sequences.

Tracking and segmentation can be challenging even in a stationary camera setting. Moving pixels can be extracted by change detection techniques such as adaptive background subtraction $[14,15]$ or frame differencing [13] when image sequences are captured by stationary cameras. The detected moving pixels are grouped into blobs according to their connectivity. Such blobs do not have object shape constraints. The extracted blobs can be merged with backgrounds or similar objects. Moreover, the appearance of an object in a video sequence tends to change due to different viewpoints and illumination changes. The variations bring trouble to these change detection techniques.

Tracking and segmentation are more difficult when video sequences are captured by a moving camera, in which the background is dynamic and hard to be modeled. Tracking and segmentation in video sequences with dynamic backgrounds have many important applica-

*E-mail: jerywangjq@yahoo.com

E-mail: yagi@am.sanken.osaka-u.ac.jp

Tel.: +81-6-6879-8422, Fax: +81-6-6877-4375

$\S$ A preliminary version of this paper appeared in Proc. of IEEE Int'l. Conf. on Robotics and Biomimetics 2007, Dec. 15 - 18, 2007, Sanya, China. tions such as wearable surveillance, mobile robots and intelligent vehicles. In this work, we do not use the stationary background assumption. Therefore our approach is applicable in sequences with dynamic backgrounds, which is important for applications in robotics. Pedestrian tracking and segmentation are particularly difficult for a number of reasons besides these mentioned above. The objects of interest appear in cluttered dynamic backgrounds and have a wide range of variations. The variations might be due to different viewpoints, human body size and poses, clothing.

In this work, tracking and segmentation are carried out consecutively. The tracking module computes bounding boxes of an object and foreground likelihood ratio images. Based on the likelihood ratio images and shape priors, the segmentation module computes the foreground segmentation results in the current frame. Since segmentation results usually contain errors, directly applying segmentation results as shape priors can mislead the consecutive segmentation. To conquer this problem, we build a shape prior set before the tracking and segmentation. The segmentation result of one frame is compared with all the shape in the shape prior set. The segmentation result, together with its nearest neighbor in the prior set, is incorporated into the segmentation of the consecutive frame.

Mean-shift algorithm is adopted in this work to do blob tracking. To deal with the problem of variations of object appearance and illuminations, we update the target models adaptively, instead of using one object model throughout video sequences. The widely used color features for the mean-shift algorithm and its variations are not always discriminative enough for target localization because illumination and viewpoint tend to change $[16,17]$. In addition, the background may have color similar to the target. We present an adaptive tracking algorithm that integrates color and shape-texture features. Good features are selected and applied to represent the target according to their descriptive ability. Since segmentation is extremely difficult especially in dynamic and clut- 
tered backgrounds, Boykov and Jolly [2] proposed an effective graph based segmentation algorithm for interactive segmentation. This algorithm and its variations have achieved excellent results in interactive segmentation [2][12] and 3D reconstruction [10]. The min-cut algorithm segments images by energy minimization. After an initialization that labels a few pixels as object and background, an energy function based on both boundary and region information is defined and minimized. The energy function consists of two terms: data term and smoothness term. The data term indicates individual labeling preference of pixels based on observed intensities and pre-specified likelihood function. Smoothness term encourage spatial coherence by penalizing discontinuities between neighboring pixels.

The traditional min-cut algorithm segments images using pixel color information. However, automatic segmentation based on color distributions alone is extremely challenging. Markov Random Fields, which are the foundation of the min-cut algorithm, provide poor prior for specific shape. Min-cut-based segmentation can give wrong shape due to ambiguous edges. It is necessary to embed shape priors into the min-cut algorithm to achieve reasonable segmentation results. Based on tracking results, shape priors are learned online and incorporated into our algorithm to alleviate the problem brought by diffuse edges or similar objects in close proximity to one another.

The remainder of the paper is organized as follows. Section 2 gives an overview of the proposed approach. Section III describes the adaptive mean-shift tracking module. Section IV presents the min-cut segmentation algorithm in which shape priors are incorporated. We evaluate the performance of the proposed method in Section V. Section VI concludes the paper.

\section{Overview}

We address object tracking and segmentation in an integrated framework in which tracking and segmentation are conducted consecutively. The input to our algorithm is a sequence of images $i: \mathscr{P} \times \mathscr{T} \rightarrow \mathfrak{R}$ for each pixel $p \in \mathscr{P}$ and each time $t \in \mathscr{T}=\{0,1,2, \ldots, T-1\}$. $i_{P} \in \Re^{T}$ denotes the temporal profile of the pixel $p$. The first frame of the sequence is initialized by labeling the target. The initialization can be done using the interactive segmentation algorithm [12]. The labeled object provides shape prior $\mathscr{S}_{0}$ for the next frame.

The tracking module computes the bounding boxes in consecutive frames using the adaptive mean-shift algorithm. The bounding boxes represent the global motion of the object.

The segmentation module computes foreground/background segmentation by minimizing the energy function considering data term smoothness term, and shape priors term. We represent the output as a labeling $f: \mathscr{P} \rightarrow \mathscr{L}$. The shape priors are learned based on the segmentation results. Our algorithm aims at finding a labeling that matches pixels of similar temporal profile while minimizing discontinuities.

\section{Adaptive multi-cue tracking}

Our tracking formulation is based on the basic mean-shift algorithm [6], with a feature selection from color and shape-texture cues. The target model is updated based on feature selection results. The adaptive tracking algorithm effectively conquers the problem brought by the target appearance variations.

\subsection{The basic mean-shift algorithm}

The mean shift algorithm is a robust non-parametric probability density estimation method for climbing density gradients to find the mode of the probability distributions of samples. It can estimate the density function directly from data without any assumptions about underlying distribution. This virtue avoids choosing a model and estimating its distribution parameters. The mean-shift algorithm has achieved considerable success in object tracking [6].

The traditional mean-shift tracker has two major drawbacks, the first of which is the appearance constancy assumption that is often violated in practical applications due to variations of viewpoints or illumination changes. We develop an adaptive mean-shift tracking algorithm in which discriminative features are selected from shape-texture and color cues. A color cue is described by color histograms and shapetexture cue is represented by gradient orientation histograms. The use of orientation histograms has been found effective in gesture recognition. It has achieved great success since the invention of Scale Invariant Feature Transformation approach [11].

Basically mean-shift algorithm is a blob tracker in which only object translation in image sequences is estimated. This is the second drawback of the basic mean-shift algorithm. It has been extended to multidimensional tracking algorithms by simultaneously estimating position, rotation [20], and scale [4]. However, few algorithms can deal with exact object segmentation. In this work, the tracking module provides global motion of the target. The exact labeling of the target is computed by the segmentation module.

\subsection{Log-Likelihood ratio images}

We compute color and gradient orientation histograms for target representation. The likelihood ratio images are computed based on the weighted histograms. The likelihood ratio produces a function that maps feature values associated with the target to positive values and those associated with the background to negative values. The frequency of the pixels that appear in a histogram bin $\left(p^{\left(b_{i n}\right)}\right)$ is calculated as $\zeta_{f}^{\left(b_{\text {in }}\right)}=p_{f}^{\left(b_{\text {in }}\right)} / n_{f}$ and $\zeta_{b}^{\left(b_{i n}\right)}=p_{b}^{\left(b_{i n}\right)} / n_{b}$, where $n_{f}$ is the pixel number of the target region and $n_{b}$ the pixel number of the background. The log-likelihood ratio of a feature value is given by

$$
L^{\left(b_{i n}\right)}=\max \left(-1, \min \left(1, \log \frac{\max \left(\zeta_{f}^{\left(b_{i n}\right)}, \delta_{L}\right)}{\max \left(\zeta_{b}^{\left(b_{\text {in }}\right)}, \delta_{L}\right)}\right)\right)
$$

where $\delta_{L}$ is a very small number ( $\delta_{L}$ is set to 0.001 in this work). The likelihood image for each feature is created by back-projecting the ratio into each pixel in the image.

\subsection{Feature selection}

We use color and texture cues for the modeling of a target. In the candidate feature set, the color cue consists of 7 different features: the color histograms of $\mathrm{R}, \mathrm{G}, \mathrm{B}, \mathrm{H}, \mathrm{S}, r$, and $g$, while the shape-texture cue consists of a gradient orientation histogram. Given $m_{d}$ features for tracking, the purpose of the feature selection module is to find the best subset feature of size $m_{m}$, and $m_{m}<m_{d}$. Feature selection can help minimize the tracking error and maximize the descriptive ability of the feature set.

We find the features with the largest corresponding variances. Based on the equality $[5] \operatorname{var}(x)=E\left[x^{2}\right]-(E[x])^{2}$, the variance of Equation 1 
is computed as

$$
\operatorname{var}(L ; p)=E\left[\left(L^{b_{i n}}\right)^{2}\right]-\left(E\left[L^{b_{i n}}\right]\right)^{2} .
$$

The variance ratio of the likelihood function is defined as [5]:

$$
\operatorname{VR}=\frac{\operatorname{var}(B \cup F)}{\operatorname{var}(F)+\operatorname{var}(B)}=\frac{\operatorname{var}\left(L ;\left(p_{f}+p_{b}\right) / 2\right)}{\operatorname{var}\left(L ; p_{f}\right)+\operatorname{var}\left(L ; p_{b}\right)} .
$$

We evaluate the discriminative ability of each feature by calculating the variance ratio. These features are ranked according to the discriminative ability by comparing the variance ratio. The feature with the maximum variance ratio is taken as the most discriminative feature.

\subsection{Target localization in adaptive tracking}

The proposed tracking algorithm combines the top two features through back-projection [3] of the joint histogram, which implicitly contains certain spatial information that is important for the target representation. We calculate the joint histogram of the target with the top two features,

$$
p_{f}^{\left(b_{i n}^{(1)}, b_{i n}^{(2)}\right)}=C \sum_{\mathbf{x}_{i} \in R_{f}} k\left(\left\|\mathbf{x}_{i}\right\|\right) \delta\left[h\left(\mathbf{x}_{i}\right)-b_{i n}^{(1)}\right] \delta\left[h\left(\mathbf{x}_{i}\right)-b_{i n}^{(2)}\right],
$$

and a joint histogram of the searching region

$$
p_{b}^{\left(b_{i n}^{(1)}, b_{i n}^{(2)}\right)}=C \sum_{\mathbf{x}_{i} \in R_{b}} k\left(\left\|\mathbf{x}_{i}\right\|\right) \delta\left[h\left(\mathbf{x}_{i}\right)-b_{i n}^{(1)}\right] \delta\left[h\left(\mathbf{x}_{i}\right)-b_{i n}^{(2)}\right] .
$$

where $h$ is histogram computed on the target and $k()$ is an Epanechnikov kernel function [6] that evaluates pixel contributions to the mode seeking.

We get a division histogram by dividing the joint histogram of the target by the joint histogram of the background,

$$
p_{d}^{\left(b_{i n}^{(1)}, b_{i n}^{(2)}\right)}=\frac{p_{f}^{\left(b_{i n}^{(1)}, b_{i n}^{(2)}\right)}}{p_{b}^{\left(b_{i n}^{(1)}, b_{i n}^{(2)}\right)}} .
$$

The division histogram is normalized for the histogram back-projection. The pixel values in the image are associated with the value of the corresponding histogram bin by histogram back-projection. The backprojection of the target histogram with any consecutive frame generates a probability image $p=\left\{p^{i}\right\}_{i=1 \ldots n_{h}}$ where the value of each pixel characterizes the probability that the input pixel belongs to the histograms. Since we are using an Epanechnikov profile the derivative of the profile, $g(x)$, is constant. The target's shift vector in the current frame is computed as

$$
\hat{\mathbf{y}}_{1}=\frac{\sum_{i=1}^{n_{h}} \mathbf{x}_{i} p^{i}}{\sum_{i=1}^{n_{h}} p^{i}} .
$$

The tracker assigns a new position to the target by using

$$
\hat{\mathbf{y}}_{1}=\frac{1}{2}\left(\hat{\mathbf{y}}_{0}+\hat{\mathbf{y}}_{1}\right) \text {. }
$$

If $\left\|\hat{\mathrm{y}}_{0}-\hat{\mathrm{y}}_{1}\right\|<\varepsilon$, this position is assigned to the target. Otherwise, let $\hat{\mathrm{y}}_{0}=\hat{\mathrm{y}}_{1}$ and compute the Equation 6 again. In our algorithm, the number of the computation is set to less than 15. In most cases, the algorithm converges in 3 to 6 loops.

\section{Shape prior}

The segmentation result of a frame probably contain errors. If we use the segmentation result as shape prior directly in the next frame, the prior can misguide the segmentation. To deal with this problem, we propose a novel strategy that combines the power of the segmentation result and shape priors built before the tracking and segmentation. For instance, in the human tracking and segmentation scenario, we build a shape prior set from precisely segmented human silhouettes in an indoor environment. These shapes in the prior set is precise. However, the shapes in this set cannot cover all the large variations of human shapes. Directly using these shapes as prior knowledge can also meet problem in practice.

In this work, we combine the power of the segmentation result of one frame and the shapes in the prior set. We compare the segmentation result with all the shapes in the prior set. The segmentation result, together with its nearest neighbor in the prior set, is incorporated into the Min-Cut algorithm.

To measure the similarity between shapes, we have to define a similarity measure. The shape similarity between two silhouettes can be measured by chamfer distance function. The measurement is especially efficient based on distance transform, which computes the distance to the nearest feature in an image. One important advantage of this measurement is the smoothness in matching shapes in different position, scale. It is insensitive to errors in shape alignment. The second advantage is its considerable tolerance to noises that are unavoidable in edge detection. It has been widely used in object recognition thanks to its properties. We use chamfer distance to find similarity between two silhouettes here.

\subsection{Chamfer distance}

Chamfer distance measures the similarity of two silhouettes which are composed of two sets of edge points. Let $S$ be a segmentation result and $C_{i}$ the set of edge points of the $i$ th shape in the prior set, chamfer distance is defined in a discrete form as

$$
d\left(S, C_{i}, \mathbf{x}\right)=\frac{1}{|S|} \sum_{\mathbf{x}_{s} \in S} \min \left\|\mathbf{x}_{S}+\mathbf{x}-\mathbf{x}_{c_{i}}\right\|,
$$

where $|S|$ denotes the number of edge points in the segmentation result $S$, and $\|\cdot\|$ the $l_{2}$ norm. The chamfer distance thus gives the mean distance of edges in $S$ to their closest edge points in $C_{i}$.

The distance calculation is expensive if each position is computed independently. The distance transform can speed up the matching process dramatically via computing the distances of the closest edge points in $E$ before the matching is carried out. Edge distance transform is defined as

$$
D=\min _{x_{e} \in E}\left\|\mathbf{x}-\mathbf{x}_{e}\right\|,
$$

Based on distance transform result, we compute chamfer distance for each position by summing the values along the edge points of $C_{i}$.

$$
d\left(S, C_{i}, \mathbf{x}\right)=\frac{1}{|S|} \sum_{\mathbf{x}_{S} \in S} D T_{E}\left(\mathbf{x}_{t}+\mathbf{x}\right)
$$

The exact Eucliden distance transform can be computed in linear time based on two-pass transform. The noises in edge detection bring errors into the distance transform results. These errors are harmful to the matching. This problem can be alleviated by truncating the distance 
transform results using a distance threshold $D_{\max }$. The truncated distance transform is defined as

$$
D T_{E}(x)=\min \left(D T_{E}(x), D_{\max }\right) .
$$

\subsection{Symmetrical similarity measure}

Online matching can be seen as traversing the tree structure of templates. Each node corresponds to matching a segmentation result with the image at some particular locations. For the locations where the distance measure between template and image is bellow a user defined threshold.

\section{Segmentation Using Shape Priors}

Boykov and Jolly [2] proposed an effective min-cut method (min$\mathrm{cut} / \mathrm{max}$-flow) for interactive segmentation based on the powerful combinatorial optimization method [9]. Segmentation is formulated in terms of energy minimization in the min-cut algorithm. The cost function is obtained in a context of Maximum A Posteriori of Markov Random Field (MAP-MRF) estimation [8]. The purpose of the min-cut/max-flow is to seek the labeling of image pixels $(\mathscr{P})$ by minimizing energy:

$$
E(L)=E_{\text {smooth }}(L)+E_{\text {data }}(L)
$$

where $L=\left(L_{1}, \ldots, L_{|P|}\right)$ is a binary vector whose components specify label assignment; $E_{\text {smooth }}$ measures the smoothness of neighboring pixels; and $E_{\text {data }}$ measures the disagreement between labeling and the observed data. $E_{\text {smooth }}$ and $E_{\text {data }}$ are formulated respectively as

$$
E_{\text {smooth }}(L)=\sum_{\{p, q\} \in \mathscr{N}} V_{p, q}\left(L_{p}, L_{q}\right)
$$

and

$$
E_{\text {data }}(L)=\sum_{p \in P} D_{p}\left(L_{p}\right)
$$

where $\mathscr{N}$ contains all unordered pairs of neighboring pixels; $V_{p, q}$ measures the smoothness of interacting pairs of pixels; $D_{p}$ is determined by the fitness of $p$ given the observed data.

\subsection{Data term}

The form for the data term requires knowledge of some information about the object and the background. In particular, suppose that we know the probability distribution over intensity for both the object and the background, the data term is computed using

$$
D_{p}(L=0)=-\log p\left(I \mid H_{b}\right),
$$

and

$$
D_{p}(L=1)=-\log p\left(I \mid H_{o}\right) .
$$

These distributions can be learned based on the segmentation results of the previous frame. We built two histogram of the color information of the target region and the background region. The data term is computed based on these distributions.

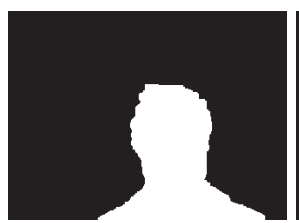

(a)

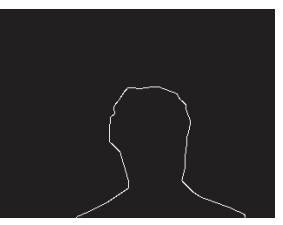

(b)

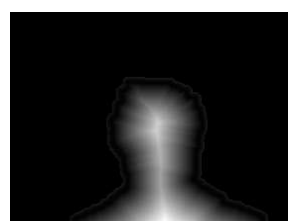

(c)
Figure 1. Generation of shape priors for the segmentation. (a) The silhouette provided by the gait recognition module; (b) the Edge detection result; and $(c)$ the Euclidean distance transform result.

\subsection{Smoothness term}

The smoothness term can be written as

$$
V=\gamma \sum_{(p, q) \in \mathscr{P}}\left[L_{p} \neq L_{q}\right] \frac{e^{-(I(p)-I(q))^{2} / 2 \sigma^{2}}}{\|p-q\|}
$$

where $[\varphi]$ denotes the indicator function taking values 0,1 for a predicate $\varphi,(p, q) \in \mathscr{P}$ is a set of pairs of neighboring pixels, and $\|p-q\|$ denotes the Euclidean distance of neighboring pixels, $\gamma$ is a coefficient for weighting of the smoothness term. This energy encourage coherence in regions of similar grey-level.

\subsection{Min-cut/max-flow segmentation with shape pri- ors}

A shape prior term is added into the energy function to embed shape priors into the min-cut/max-flow algorithm. The energy function now is defined as

$$
E(L)=E_{\text {smooth }}(L)+E_{\text {data }}(L)+E_{\text {shape }}(L) .
$$

The shape priors is obtained based on segmentation results of the previous frame. The min-cut now includes the shape fitness as well as smoothness and data initial labeling. The energy function $E_{\text {shape }}$ is penalized if the segmented contour deviates from the boundary of the silhouette.

The shape prior can be represented by a distance transform result[7]. In Figure 1, edges are detected in the silhouette image using Canny edge detector. Then the Euclidean distance transformation [1] of the edge image is computed. The cost function of shape priors is well described in the transformed image where costs depend on the distance from the edges. The shape prior energy is written as

$$
E_{\text {shape }}=\sum_{(p, q) \in \mathscr{N}}\left[L_{p} \neq L_{q}\right] \frac{\psi(p)+\psi(q)}{2}
$$

where $\psi$ is a value on the transformed image.

Segmentation is achieved by minimizing energy function described in Equation 12. 

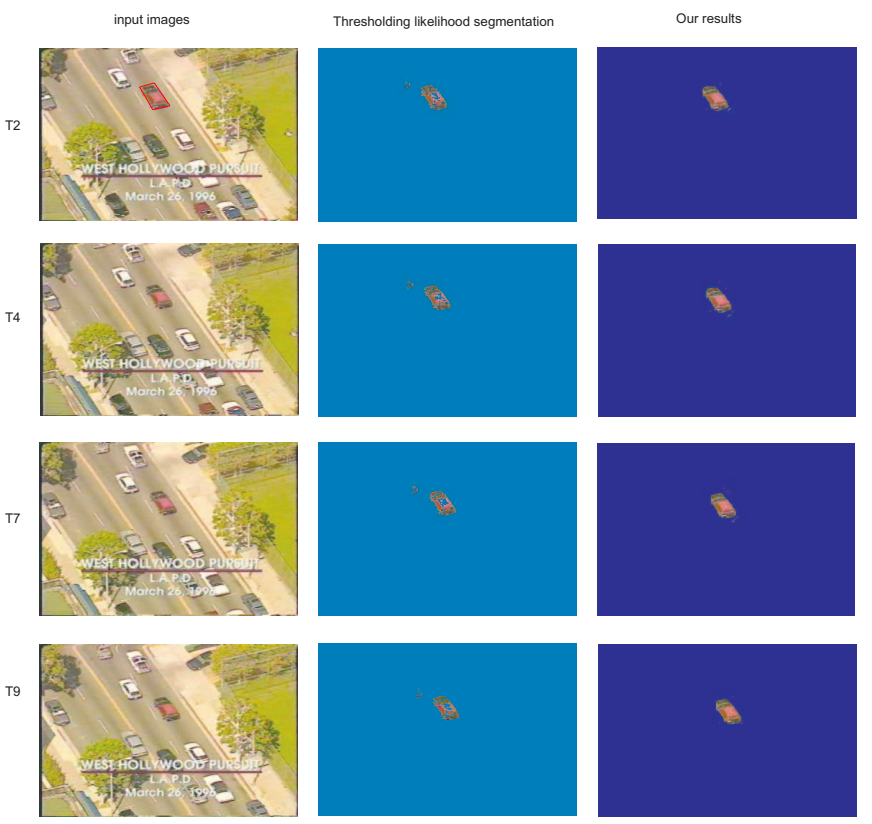

Figure 2. Comparison of our results on the car sequence with other methods: Left: input images; Middle: Results from segmentation of the manually selected difference image. Right: Results from our algorithm. The camera is not stationary in the sequence.

\section{Experimental results}

The proposed object tracking and segmentation algorithm is applied to a variety of sequences to verify the performance. The proposed approach is compared with segmentation by thresholding likelihood ratio images. Given a threshold, likelihood ratio images are transformed into binary images where target and its background are labeled. This is an efficient method that has been used in tracking for target modeling. We have applied our approach on many image sequences. Two of them are showed in Figure 2 and Figure 3 respectively. We show the segmentation results and mixing results in Figure 4.

The car sequence is captured by a moving camera. Hence there are consistent motions for both foreground and background, which may cause trouble for both pixel-wise background subtraction [14] and layer-based motion segmentation approaches [15]. Our algorithm is not affected by such motions. The segmented region using our algorithm provides coherent region and boundaries of the car. In contrast, the results from the thresholding likelihood ratio method are not satisfying because there are many foreground pixels are labeled as background. The comparison demonstrates that our approach outperforms the thresholding likelihood ratio method.

The person talking sequence is captured in an indoor environment in which a person is talking while moving his body freely. The segmentation results can be useful in human computer interface and teleconference. In Figure 3, the proposed approach provides better segmentation results. However, there are some pixels are mislabeled by our approach (especially in $T 80$ and $T 98$ ). The reason is that the proposed approach suffers from "pollution" of shape priors. When the shape priors computed by the segmentation module deviate from the true shape, it can accumulate and misguide the segmentation. One possible solution to this problem is to learn accurate object shapes offline and apply the
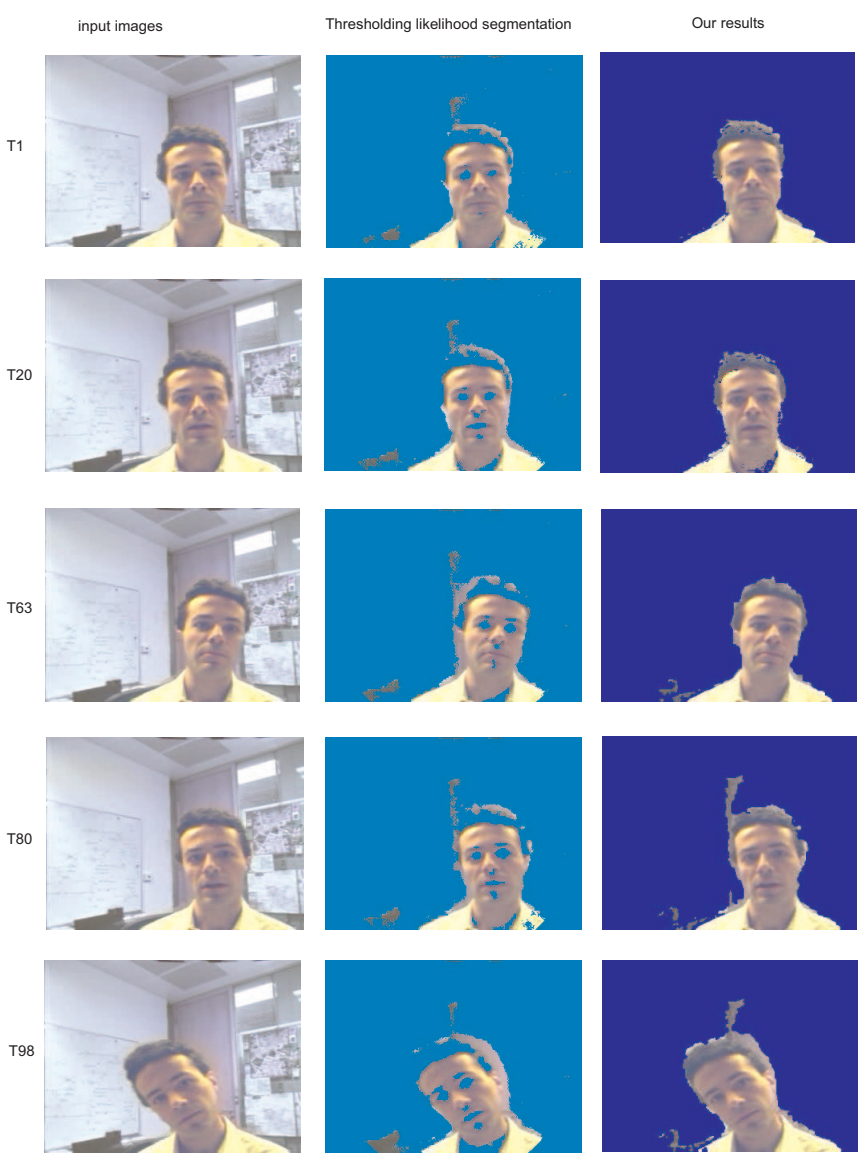

Figure 3. Comparison of our results on the person talking image sequence with other methods: Left: input images; Middle: Results from segmentaother methods: Left: input images; Middle: Results from segmenta-
tion of the manually selected difference image. Right: Results from our algorithm.

learned priors to specific frames.

The person walking and talking sequence is captured in an indoor environment by a moving camera. In the dynamic background, a person is walking in front of a blackboard. We mix the segmentation results with another background. Figure 4 shows the mixed results. In the mixed results, the segmented person is scaled and repositioned in the new background. The results indicate that the proposed algorithm is applicable to computer graphics related to robotics.

To show the effectiveness of our method, we give the ground truth of the person talking sequence by marking the images manually. The segmentation errors of our method and of the binarized likelihood image. The comparison in Figure 5 demonstrates that our method has better performance than the results derived using the likelihood image. We additionally note that we ran our algorithm on these sequences without any parameter tuning.

Timing The proposed algorithm is efficient. When it is applied to the car sequence (images of size $360 \times 240$, the tracking module spends 0.03 seconds for each frame and the segmentation module uses $0.09 \mathrm{sec}-$ onds. The segmentation in the man sequence is a little more expensive than that in the car sequence since the target is much larger. It takes 0.19 seconds to segment the man. These experiments demonstrate 
(a)
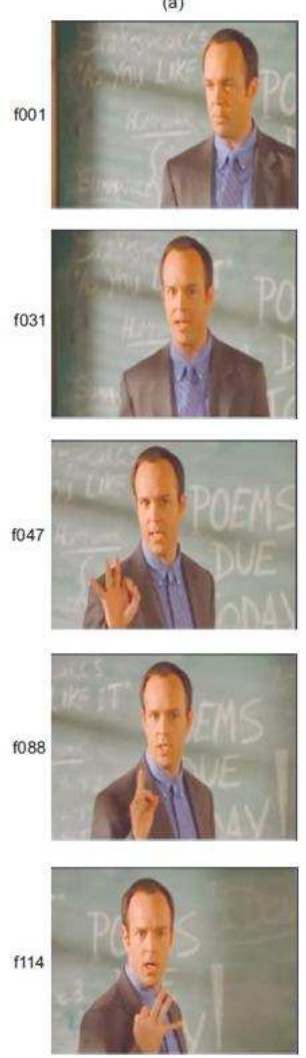

(b)
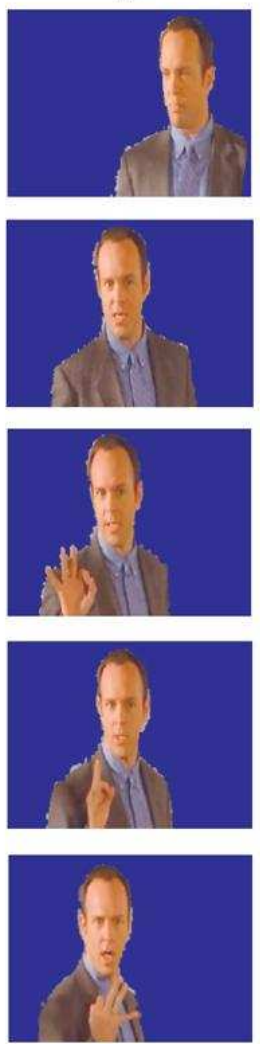

(c)
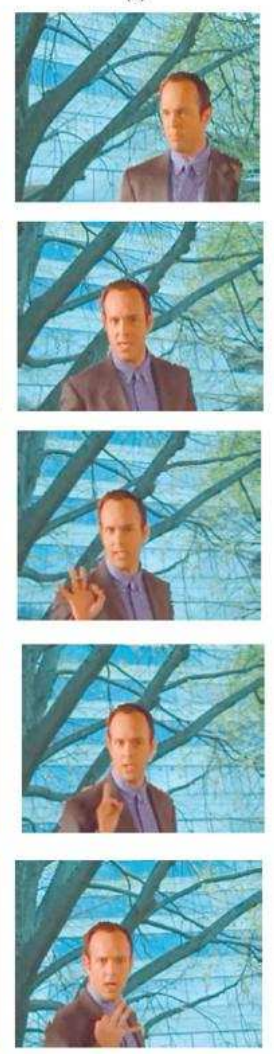

Figure 4. The segmentation and mixing results of a walking person. Left: the input images; Middle: segmentation results using the proposed algorithm. Right: mixing the segmentation results with another background.

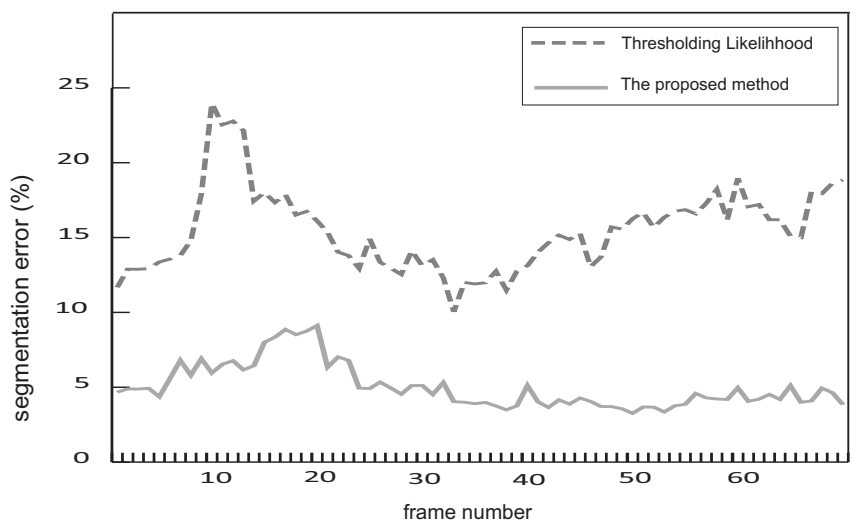

Figure 5. Quantitative Analysis of the sequence shown in Figure 2. The proposed method has better performance than other approach in most of the frames.

that our algorithm is efficient and effective. All the tests are conducted on an Intel Centrino 1.6GHz laptop with 1GB RAM.

The processing time for tracking and segmentation will increase when the size of the target is larger. The computational cost of the tracking module is sub-linear to the size of the target since we use a probability density gradient-based searching strategy. The computational cost of the segmentation module is more complicated. The maximum flow from the source to the sink node is an upper bound on the number of augmenting paths. Fortunately, the cost is a polynomial complexity in total. The investigation of further efficiency improvement is one of the important future works.

\section{Conclusion and future work}

We have devised an object tracking and segmentation algorithm that compute the global movement and local refinement. The global movement of an object is given by the tracking module and the min-cut segmentation module computes the detail object segmentation. Shape priors are learned based on the tracking and segmentation results. They are incorporated into the min-cut algorithm, which improves the segmentation performance.

It is interesting to investigate how to use shape priors efficiently and effectively when shape variations are large. In addition to the proposed method, actions with different silhouettes can be tracked and classified using shape information. We are working on concurrent classification and segmentation.

\section{References}

[1] A. Boregefors. "Distance transformations in digital images", Computer Vision, Graphics and Image Processing, Vol. 34(3), pp. 344371, 1986.

[2] Y. Boykov and M-P. Jolly. "Interactive graph cuts for optimal boundary and region segmentation of objects in n-d images," in Proc. of Int'I Conf. on Computer Vision, pp. 105-112, 2001.

[3] G.R. Bradski, "Computer Vision Face Tracking as a Component of a Perceptural User Interface," in Proc. of the IEEE Workshop Applications of Computer Vision, pp. 214-219, 1998.

[4] R. T. Collins, "Mean-shift Blob Tracking through Scale Space," in Proc. of the IEEE Conf. on Computer Vision and Pattern Recognition, pp. 234-240, 2003.

[5] R. T. Collins and Y. Liu, "On-line Selection of Discriminative Tracking Features," in IEEE Trans. on Pattern Analysis and Machine Intelligence, Vol. 27, No. 10, October 2005.

[6] D. Comaniciu, V. Ramesh, and P. Meer, "Kernel-based Object Tracking," IEEE Trans. Pattern Analysis Machine Intelligence, vol. 25, no. 5, pp. 564-577, 2003.

[7] D. Freedman and T. Zhang. "Interactive graph cut based segmentation with shape priors," in Proc. of IEEE Conf. on Computer Vision and Patten recognition, pp. 755-762, 2004.

[8] S. Geman and D. Geman. "Stochastic relaxation, Gibbs distributions, and the Bayesian restoration of images." IEEE Transactions on Pattern Analysis and Machine Intelligence, 6:721C741, 1984.

[9] D. Greig, B. Porteous, and A. Seheult. "Exact maximum a posteriori estimation for binary images". Journal of the Royal Statistical Society, Series B, 51(2):271C279, 1989.

[10] V. Kolmogorov and R. Zabih, "Multi-camera Scene Reconstruction via Graph Cuts", in Proc. the 7th European Conference on Computer Vision (ECCV 02), May, 2002. 
[11] D. G. Lowe. Distinctive image features from scale-invariant keypoints. International Journal of Computer Vision, vol. 60, no. 2 pp. 91-110, 2004.

[12] C. Rother, V. Kolmogorov, and A. Blake, GrabCut -Interactive Foreground Extraction using Iterated Graph Cuts, ACM Transactions on Graphics (SIGGRAPH), August 2004.

[13] A. Rowe, C. Rosenberg, and I. Nourbakhsh, "A second generation low cost embedded color vision system," in Workshop in Proc. of the IEEE Conf. on Computer Vision and Pattern Recognition (CVPRW'05), pp. 136, 2005.

[14] C. Stauffer and E. Grimson, "Learning pattern of activity using realtime tracking," IEEE Trans. on Pattern Analysis and Machine Intelligence, vol. 22, no. 8, pp. 747-757, 2000.

[15] H. Tao, H. S. Sayhney, and R. Kumar, "Dynamic Layer Representation with Applications to Tracking," in Proc. of the IEEE Conf. on Computer Vision and Patter Recognition, vol. 2, pp. 134-141, 2000.
[16] J. Wang and Y. Yagi. "Integrating shape and color features for adaptive real-time object tracking", 2006 IEEE Int'l Conf. on Robotics and Biomimetics, 2006.

[17] J. Wang and Y. Yagi. "Consecutive tracking and segmentation using graph-cut", 2007 IEEE Int'I Conf. on Robotics and Biomimetics, 2007.

[18] J. Wang and Y. Yagi. Integrating Color and Shape-texture Features for Adaptive Real-time Tracking, IEEE Trans. on Image Processing, 17(2): 235-240, (2008).

[19] J. Wang, Y. Makihara, and Y. Yagi. "People Tracking and Segmentation Using Spatiotemporal Shape Constraints", In Proc. of 1st ACM International Workshop on Vision Networks for Behaviour Analysis, Vancouver, Canada, Oct. 31, 2008.

[20] Z. Zivkovic and B. J. A. Krose, "An EM-Like Algorithm for ColorHistogram-Based Object Tracking," in Proc. of the IEEE Conf. on Computer Vision and Patter Recognition, pp. 798-803, 2005. 